\title{
Effectiveness of Interventions to Prevent Sexually Transmitted Infections and Human Immunodeficiency Virus in Heterosexual Men
}

\author{
A Systematic Review
}

A. Rani Elwy, PhD; Graham J. Hart, PhD; Sarah Hawkes, PhD, MBBS; Mark Petticrew, PhD

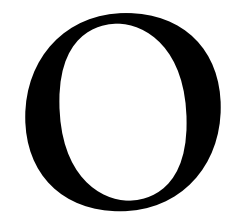

ur objective was to review systematically studies of interventions to prevent transmission of sexually transmitted infections (STIs) and human immunodeficiency virus (HIV) in heterosexual men. Of 1157 studies identified through database and hand searching, 27 met our inclusion criteria. Most interventions targeted specific groups of men (eg, those attending STI clinics) rather than general populations. Few were conducted with men alone, and most focused on behavioral and social psychological rather than morbidity outcomes. Of 8 interventions designed to reduce STI incidence (including HIV), 5 were successful, 2 were unsuccessful, and 1 had equivocal results. Of the 5 successful interventions, 1 was carried out in the workplace, 1 in the military, and 3 in STI clinics. They included on-site individual counseling and HIV testing, mass communications regarding risk reduction, and multiple-component motivation and skills education in STI clinics. More high-quality research into the effectiveness of interventions targeting heterosexual men is needed, especially methodologically sound trials to evaluate effects on morbidity.

Arch Intern Med. 2002;162:1818-1830

Heterosexual transmission of human immunodeficiency virus (HIV) is increasing globally. ${ }^{1}$ This is despite widespread efforts to prevent the spread of the disease through behavioral and educational interventions and delivery of services aimed at offering free HIV testing and counseling. Additionally, treatment for sexually transmitted infections (STIs) known to facilitate HIV transmission has also been used to control its spread among men and women. ${ }^{2,3}$ Heterosexual men may be key to controlling the spread of the STI and HIV epidemics. Sexually transmitted infections, including HIV, are more easily transmitted from men to women than women to men. ${ }^{4}$ Indeed, women are twice as likely as men to become infected by a variety of sexually transmitted pathogens, ${ }^{5}$ and the efficiency of male-tofemale transmission of HIV is approximately 4 times higher than female-tomale transmission. ${ }^{6}$ Aside from the

From the Medical Research Council, Social and Public Health Sciences Unit, University of Glasgow, Glasgow, Scotland (Drs Elwy, Hart, and Petticrew), and the London School of Hygiene and Tropical Medicine, London, England (Dr Hawkes). increased biological risk of transmission, women may be at high risk of STI and HIV owing to social and cultural norms of behavior that dictate that women cannot decline sexual intercourse with their partners or insist on the use of barrier methods for protection during intercourse. ${ }^{7}$ Moreover, these same social and cultural norms often allow men to seek sexual pleasure outside of the home, thereby possibly increasing the risk of acquiring STIs, including HIV. ${ }^{8}$

No systematic reviews have been conducted to determine the most effective social and behavioral means of preventing the spread of STIs and HIV among heterosexual men. Reviews conducted on populations other than heterosexual men have demonstrated that few studies are of sound methodological quality. In a review of 68 behavioral interventions to prevent HIV and acquired immunodeficiency syndrome (AIDS), ${ }^{9}$ the authors rated only 18 of these as being of high methodological quality. Five of the 18 interventions were judged to be effective at preventing HIV and AIDS by the reviewers, while 7 were judged ef- 
Table 1. Databases and Years Searched

\begin{tabular}{lc}
\hline Database & Years Searched \\
\hline AIDSLine & $1980-2000$ \\
ASSIA & $1987-1999$ \\
BIDS (IBSS; EMBASE) & $1980-1999$ \\
British Nursing Index (BNI) & $1985-1998$ \\
CINAHL & $1982-1999$ \\
Cochrane Database of Systematic Reviews & $1998-2000$ \\
Current Research in Britain (CRIB) & 199913 th ed \\
Database of Reviews of Effectiveness (DARE) & $1995-1999$ \\
ERIC & $1966-1999$ \\
HealthPromis (Health Development Agency, United Kingdom) & $1964-2000$ \\
HealthSTAR & $1988-1999$ \\
Health CD & $1983-1999$ \\
Helping Involve Men (HIM; Johns Hopkins) & $1983-2000$ \\
HMIC (King's Fund, United Kingdom) & $1979-2000$ \\
MEDLINE & $1966-2001$ \\
National Research Register (United Kingdom) & $1998-1999$ \\
OCLC PapersFirst \& Proceedings First & $1993-1999$ \\
Popline & $1983-1999$ \\
Psyclnfo & $1967-2000$ \\
SIGLE & $1980-2000$ \\
Sociological Abstracts & $1963-2000$ \\
&
\end{tabular}

fective by the authors of the studies. Four of these 5 effective interventions demonstrated a change in young people's sexual behavior, while the remaining effective intervention targeted homosexual and bisexual men.

Systematic reviews of interventions that consist of providing information on the HIV status of participants in an effort to prevent transmission have also been undertaken. In a meta-analysis of $27 \mathrm{HIV}$ testing and counseling interventions, ${ }^{10}$ it was concluded that HIV counseling and testing was not an effective primary prevention strategy for uninfected participants, although it seemed to provide an effective means of secondary prevention for HIV-positive individuals. However, heterosexual men were not the main focus of these reviews. Men are not a homogeneous group. ${ }^{11} \mathrm{Het}-$ erosexual men of all ages may have different needs than women, men who have sex with men, or adolescents as a group and, based on prevailing attitudes and sociocultural and demographic characteristics, they may also respond differently than these other populations to interventions. Therefore results from existing systematic reviews cannot necessarily be generalized to adult men who have sex with women.

A second limitation of previous systematic reviews assessing the effectiveness of interventions to prevent STIs and HIV is that they only sought to include North American studies. It is known that men's attitudes toward contraception affect efforts to prevent STIs in the United States. ${ }^{12}$ However, the association between men's sexual attitudes and their sexual behavior in other parts of the world has not been reviewed systematically. The spread of HIV is currently most rapid in other parts of the world, including the countries of the former Soviet Union and Asia. ${ }^{1}$ Interventions targeting North American populations, while important, will not substantially reduce the global threat of STI and HIV.

Finally, few reviews in this area have assessed the effectiveness of interventions to prevent the spread of STIs, along with HIV, in heterosexual men. Given that STIs can facilitate the acquisition and transmission of $\mathrm{HIV}^{13}$ and efforts to prevent the spread of HIV through treatment of STIs in women and men have proven effective,${ }^{14}$ it is imperative that systematic reviews to determine the effectiveness of interventions to prevent HIV infection include interventions targeting STIs. The present systematic review takes account of the above limitations of previous reviews and seeks to determine the most effective social and behavioral means of preventing the
Table 2. MEDLINE Search Strategy: Key Terms Used

\begin{tabular}{ll} 
1. & Men/ \\
2. & Male/ \\
3. & 1 or 2 \\
4. & Sexually transmitted diseases/ \\
5. & Gonorrhea/ \\
6. & Chlamydia/ \\
7. & Syphilis/ \\
8. & Genital herpes/ \\
9. & Condylomata acuminata/ \\
10. & Urogenital diseases/ \\
11. & 5 or 6 or 7 or 8 or 9 or 10 or 11 \\
12. & 4 and 11 \\
13. & 3 and 12 \\
14. & Sex behavior/ \\
15. & 3 and 4 \\
16. & HIV/ \\
17. & 3 and 16 (not 12 ) \\
18. & Prevention/ \\
19. & 12 and 18 \\
20. & 3 and 19 \\
21. & Community health/ \\
22. & 3 and 21 \\
23. & Health services/ \\
24. & 3 and 23 \\
25. & Reproductive health/ \\
26. & 3 and 25 \\
27. & 26 not 24 \\
28. & 26 not 22 \\
& \\
\hline 20 &
\end{tabular}

spread of HIV and other STIs in heterosexual men.

\section{METHODS}

\section{AIM AND RESEARCH QUESTION}

We undertook a systematic review to evaluate the evidence for the effectiveness of social and behavioral interventions to promote men's sexual and reproductive health, focusing on the prevention of STIs including HIV. To this end, we sought to anwer the question, "Which interventions are successful in reducing the transmission of STIs and HIV in heterosexual men?"

\section{SEARCH STRATEGY}

A list of the databases searched is given in Table 1. Key words used in the MEDLINE search are listed in Table 2. These were adapted for use in the other database search strategies. Additional search strategies included hand searching 4 key journals (AIDS, AIDS Care, International Journal of STD and AIDS, and Sexually Transmitted Diseases); hand searching references of all studies meeting the reference criteria; and writing to the first authors of all studies that met the inclusion criteria to request information on unpublished work or research in progress. 


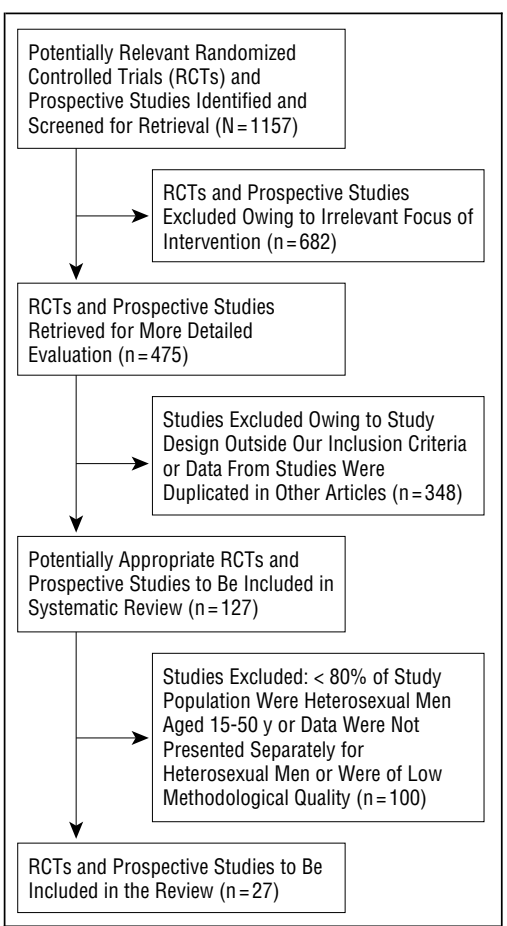

Selection of interventions for systematic review.

Studies located by the search strategy were coded for inclusion using a checklist. The reliability of this checklist was tested by 2 of the authors on a subsample of 25 studies. The Cohen $\kappa$ for this reliability was 0.91 , considered a very high interrater agreement. ${ }^{15}$ One author coded the remaining studies for inclusion.

\section{INCLUSION CRITERIA}

Studies were selected for review if they met the following 5 criteria: (1) intervention populations included heterosexual male subjects 15 years or older; (2) data on heterosexual men's sexual behavior were presented and analyzed separately from other groups included in the study or at least $80 \%$ of the study participants were heterosexual men; (3) study designs were coded in the following categories: (i) randomized or nonrandomized controlled, (ii) prospective observational, or (iii) retrospective observational; (4) the outcomes assessed in the study included at least 1 of the following: (i) morbidity (new or reinfection with STI, including HIV), (ii) behavioral outcomes (eg, condom use or reduction in the number of sex partners), and (iii) social psychological outcomes (eg, attitudes toward condoms or HIV or intentions to use condoms); and (5) study was coded as being of moderate to high methodological quality. Unpublished studies and studies in all languages were considered for inclusion in the review.

\section{QUALITY ASSESSMENT}

Two authors independently assessed the methodological quality of the studies meeting the first 4 inclusion criteria by using 1 of 2 checklists. For behavioral studies using a randomized controlled trial (RCT) or prospective-observational design with more than 1 study group, an adapted version of a quality checklist standardized by Jadad et $\mathrm{al}^{16}$ was used. We adapted criterion 2 of the original checklist to read "Was this study single blind?" instead of "double blind" and assigned a maximum score of 4 to each study, with higher scores denoting higher methodological quality. For studies using a prospective design with only 1 study group (before-and-after studies) or a retrospective observational design, we used a methodological quality checklist with a maximum quality score of $5 .{ }^{17}$ Both quality checklists included items consistent with the consensus statement of meta-analysis reporting of observational studies in epidemiology. ${ }^{18}$ Differences in ratings between the authors were reconciled through discussion, and consensus was reached. Studies that received a quality score of 1 or lower based on these checklists were excluded from the review.

\section{DATA COLLECTION}

Data from studies meeting the inclusion criteria were extracted using a checklist. ${ }^{17}$ Two authors pilot-tested this checklist prior to its use in the study, and disagreements in data extraction were resolved through discussion. Study information and data were recorded in the Microsoft Access database.

\section{RESULTS}

INTERVENTION CHARACTERISTICS

Of the 1157 articles located through hand searching and electronic databases, 27 studies met the inclusion criteria (Figure). No additional studies were located through contact with experts in the field. Twelve (44\%) of the studies were conducted on male-only populations. Heterosexual men in the 27 studies tended to fall into 5 welldefined populations: drug users receiving treatment $(3 / 27,11 \%)$; injecting drug users out of treatment $(2 / 27,7 \%)$; patients of sexually transmitted disease clinics (9/27, $33 \%)$; men in the workplace $(3 / 27$, $11 \%)$; and students $(6 / 27,22 \%)$.
Other populations of men included (in 1 study each) were African American men via outreach (ie, not attending clinic); prisoners; homeless men with psychiatric problems; and military men.

Women were included in 15 (56\%) of the studies. In 8 of these, the interventions addressed men and women in different groups, or men and women were given individual intervention sessions; in the other 7 studies, women and men were addressed together in groups.

Most studies were North American, with 17 (63\%) conducted in the United States. Eight (30\%) of the 27 studies specifically targeted racial and ethnic minorities in US populations. Two studies were undertaken in Brazil, and 1 study each was conducted in Britain, Australia, India, Kenya, Mozambique, Namibia, Senegal, and Thailand.

Twenty studies included in the review were RCTs, and the other 7 were of a prospective experimental design. Six of these studies did not have a control or a comparison group. ${ }^{19-24}$ No retrospective observational interventions were included in the review.

Eight studies (30\%) evaluated morbidity outcomes (new HIV infection or new or reinfection of STI); 21 (78\%) assessed behavioral outcomes (condom use, reduction in number of sex partners, and unprotected sex); and 15 (56\%) assessed social psychological outcomes (attitudes toward condoms or HIV, intentions to use condoms or change risky behavior, knowledge of HIV and AIDS, self-efficacy of condom use, communication skills, and quality of sexual relationships).

The interventions identified by the present review are either groupbased or individual interventions with heterosexual men (and sometimes women). Group-based interventions tended to be weekly 1 - to 2-hour sessions over 2 to 4 weeks. These sessions involved exclusively male groups or men and women together, and they were conducted by trained facilitators, either professionals or peer educators. Usually, the goal of these sessions was to change sexual risk behavior through education, communication skills training, and role- 
playing. Condoms were often distributed free of charge during these sessions, and condom use skills were taught.

Individually based intervention sessions tended to consist of individual counseling with a trained professional or peer educator. These sessions were aimed at either men alone or at men and women, but men and women did not meet with the counselor together. The counselor offered pre-HIV test counseling, confidential HIV testing, posttest counseling, a personalized evaluation of risk for STI and HIV, and/or a personalized risk reduction plan. Further education could be offered to the client, and condoms were often distributed free of charge.

\section{INTERVENTIONS BY GROUP OF MEN}

\section{Drug Users Receiving Treatment}

All included sources, ${ }^{19-45}$ categorized according to groups of men, are listed in Table 3. Three studies of moderate quality were carried out with heterosexual men receiving treatment for drug use. Two of these studies were RCTs, ${ }^{25,26}$ and 1 used a prospective uncontrolled design. ${ }^{22}$ Two interventions aimed to increase condom use, ${ }^{22,25}$ and 1 aimed to decrease the number of sex partners of these men. ${ }^{26}$ These interventions were highly successful at changing the sexual behavior of this group of men. Both interventions that aimed to increase condom use in men reported a significant intervention effect in the experimental group. ${ }^{22,25}$ A decrease in the number of sex partners was reported for the study that aimed to change this behavior. ${ }^{26}$ Interventions that reported a significant effect on behavioral outcomes varied in their design. Two interventions featured educational and motivational aspects and focused on risk prevention, increasing levels of personal concern regarding HIV/AIDS, and personalizing the threat of HIV. ${ }^{25,26}$ These interventions also addressed the skills needed for negotiating condom use and preventing a relapse in drug use and risky sexual behavior, with men participating in separate intervention sessions from wom- $e^{25}$ or in a group exclusively of men. ${ }^{26}$ One intervention that was successful in increasing condom use involved a condom giveaway program in a drug abuse outpatient treatment clinic. ${ }^{22}$ There were no educational or cognitive-behavioral elements in this intervention. One intervention with drug users receiving treatment also focused on social psychological outcomes: Malow et $\mathrm{al}^{26}$ reported increases in knowledge of HIV, condom use skills, sexual communication skills, and response efficacy for using condoms in the intervention and control groups.

\section{Out-of-Treatment Injecting Drug Users}

Two moderate- to high-quality studies aimed to increase use of condoms among a groups of heterosexual men, ${ }^{27,28}$ and 1 study also aimed to decrease numbers of sexual partners. ${ }^{27}$ Both studies used RCT designs. Cottler et $\mathrm{al}^{27}$ conducted drug awareness, stress management, AIDS education, and personal risk awareness sessions through a peereducation outreach program, using a storefront satellite to recruit men. The authors report that no change in condom use or number of sexual partners was reported as a result of the intervention. Condom use did increase in the study by Robles et al. ${ }^{28}$ This intervention consisted of 8 sessions designed to improve individuals' perceptions of risk and provide motivation to change risk behavior. Risk behavior was monitored over 6 months of follow-up. A control group received 2 sessions of standard care including HIV pretest and posttest counseling. The reported increase in condom use was seen in the intervention and control groups.

\section{Men in the Workplace}

Three moderate-quality studies of a prospective uncontrolled design were carried out with heterosexual men in the workplace. ${ }^{19,23,24}$ One study, which consisted of an intervention with trucking workers in Kenya, reported a significant intervention effect on lowering the STI incidence and decreasing the number of sex partners of these men. ${ }^{23}$ An in- crease in condom use was not reported for this cohort. The intervention comprised on-site counseling and HIV testing. Skills in condom negotiation, condom use demonstration and promotion, and STI and HIV risk reduction were provided in individual sessions with the men.

The intervention evaluated by Hearst et al $^{19}$ aimed to change the sexual behavior and attitudes toward condoms of Brazilian port workers and to increase their knowledge of AIDS using face-to-face contact by peer outreach workers to discuss HIV and AIDS, either individually or in groups, and free distribution of condoms. The authors reported a significant increase in reported condom use and a decrease in the number of sex partners of the men over a 2-year follow-up period. However, no change was reported in the men's attitudes toward condoms or their knowledge of HIV and AIDS.

In a peer-education intervention with trucking workers in Senegal, significant effects were reported over 2 years of study in increasing men's condom use and HIV knowledge and in eliminating perceived barriers to changing sexual behavior. ${ }^{24}$ The peer educators, initially part of the intervention group and subsequently selected and trained as peer educators by the authors, gave the workers information about HIV and STDs, including mode of transmission, signs and symptoms, and methods of prevention. Skills related to condom use, condom negotiation, and communication were all taught. Reports from female sex workers frequented by the men corroborated the reports of men's use of condoms. However, reports of men having fewer sex partners after intervention were not corroborated by the women. The intervention effect on the men's reports of decreases in the number of sex partners was determined unreliable by the authors.

\section{Men Attending STI Clinics}

Nine moderate- to high-quality studies focused on preventing the spread of STIs and HIV in men attending public STI clinics. ${ }^{21,29-36}$ Interventions with this group of men were 
Table 3. Characteristics, Results, and Assessed Quality of Included Sources*

\begin{tabular}{|c|c|c|c|c|}
\hline $\begin{array}{l}\text { Source; } \\
\text { Country; I }\end{array}$ & Design; Outcomes & Description & Results & $\begin{array}{c}\text { Assessed } \\
\text { Quality }^{16}\end{array}$ \\
\hline $\begin{array}{l}\text { Calsyn et al }{ }^{22} \text { (1992); } \\
\text { United States } \\
\text { (Washington State); } \\
\text { condom giveaway } \\
\text { program }\end{array}$ & $\begin{array}{l}\text { Prospective, } 1 \text { group; } \\
\text { condom use }(+) \text {, } \\
\text { intention to use } \\
\text { condoms }(+) \text { (condom } \\
\text { possession) }\end{array}$ & $\begin{array}{l}\text { Drug Users in Treatment } \\
\text { Men receiving outpatient drug-abuse } \\
\text { treatment and remaining in treatment } \\
\text { for } 4 \text { mos. I: placing jars with } \\
\text { individually packaged condoms in } \\
\text { staff offices and public rooms. } \\
\mathrm{N}=103 \text {. Follow-up: } 4 \text { mo. }\end{array}$ & $\begin{array}{l}56 \text { clients }(60 \%) \text { reported taking condoms during the } \\
\text { I. The number of clients possessing condoms } \\
\text { increased from } 61(59 \%) \text { initially to } 71(76 \%) \text { at } \\
\text { follow-up (binomial } P<.05) \text {. The mean use of } \\
\text { condoms for vaginal sex increased from } 20 \% \text { to } \\
33.7 \% \text { at follow-up }(P=.05) \text {. More clients }(36.5 \%) \\
\text { reported using condoms for at least } 50 \% \text { of vaginal } \\
\text { intercourse events in the previous } 2 \text { mo at } \\
\text { follow-up than at initial assessment (binomial } \\
P<.05) \text {. }\end{array}$ & $3 / 5$ \\
\hline $\begin{array}{l}\text { Baker et al }{ }^{25} \text { (1994); } \\
\text { Australia; motivational } \\
\text { interview to reduce } \\
\text { HIV risk-taking } \\
\text { behavior among IDUs }\end{array}$ & $\begin{array}{l}\text { RCT; HIV risk-taking } \\
\text { behavior (++) } \\
\text { (condom use, number } \\
\text { of sex partners) }\end{array}$ & $\begin{array}{l}\text { Out-of-treatment IDUs. I: } 1 \text { motivational } \\
\text { interview and a self-help booklet. } \\
\text { C: no I. } N=159 \text { men. Numbers for } \\
\text { men in I and C groups not reported. } \\
\text { Follow-up: } 3 \text { and } 6 \text { mo. }\end{array}$ & $\begin{array}{l}\text { No change in sexual risk-taking behavior from } \\
\text { pretreatment to follow-up for men in the I and } C \\
\text { groups at } 3 \text { mo ( } 3.60 \text { vs } 3.46 ; P>.05 \text { ) or } 6 \text { mo } \\
\text { (3.24 vs } 3.0, P>.05) \text {. No group differences } \\
\text { between I and } C \text { groups on any measure at } 3 \text { - or } \\
\text { 6-mo follow-ups (all comparisons, } F<1 \text { ). }\end{array}$ & $2 / 4$ \\
\hline $\begin{array}{l}\text { Malow et al }{ }^{26} \text { (1994); } \\
\text { United States } \\
\text { (Louisiana); culturally } \\
\text { sensitive HIV } \\
\text { risk-reduction Is } \\
\text { within drug-abuse } \\
\text { treatment program }\end{array}$ & $\begin{array}{l}\text { RCT; sexual risk behavior } \\
(++), \text { number of sexual } \\
\text { partners }(+), \text { HIV } \\
\text { knowledge }(++) \\
\text { response efficacy }(++) \text {, } \\
\text { sexual communication } \\
\text { skills }(++), \text { condom } \\
\text { use skills }(++)\end{array}$ & $\begin{array}{l}\text { Cocaine-using black men. I: focus on } \\
\text { developing rapport, personalizing HIV } \\
\text { threat, condom use, barriers to } \\
\text { change, skills. C: same as I, but } \\
\text { delivered as audiovisuals and printed } \\
\text { material. I: } \mathrm{n}=76 ; \mathrm{C}: \mathrm{n}=76 \\
\text { (baseline). Follow-up: post-I and at } \\
3 \text { mo. }\end{array}$ & $\begin{array}{l}\text { Rates of multiple partners fell from } 75.5 \% \text { to } 47.5 \% \\
\text { at } 3 \text { mo (binomial } P<.001 \text { ) in I group. No } \\
\text { difference in } C \text { group. No group differences in } \\
\text { knowledge, response efficacy, communication } \\
\text { skills, or condom use skills. Higher self-efficacy } \\
\text { (3.94 vs } 3.52 ; P<.05 \text { ), sexual communication } \\
\text { skills ( } 3.87 \text { vs } 3.37 ; P<.01 \text { ), and condom use skills } \\
\text { (5.49 vs } 4.37, P<.001 \text { ) in I group. }\end{array}$ & $2 / 4$ \\
\hline \multicolumn{5}{|c|}{ Out-of-Treatment Injecting Drug Users } \\
\hline $\begin{array}{l}\text { Cottler et al }{ }^{27} \text { (1998); } \\
\text { United States } \\
\text { (Missouri); } \\
\text { peer-delivered } \\
\text { enhanced I vs } \\
\text { standard I for reducing } \\
\text { HIV risk behaviors }\end{array}$ & $\begin{array}{l}\text { RCT; condom use }(-) \text {, } \\
\text { number of sex } \\
\text { partners }(-)\end{array}$ & $\begin{array}{l}\text { Out-of-treatment drug users. I: } 4 \\
\text { peer-oriented sessions on drug } \\
\text { awareness, stress management, AIDS } \\
\text { information, and reducing high-risk } \\
\text { sexual behavior. C: } 2 \text { standard } \\
\text { sessions. I: } n=246 \text { men; C: } n=196 \\
\text { men (baseline). Follow-up: } 3 \text { mo. }\end{array}$ & $\begin{array}{l}\text { No group differences in the change in number of sex } \\
\text { partners }(I=75 \% \text { vs } C=75 \% ; P>.05) \text { or condom } \\
\text { use }(I=47 \% \text { vs } C=46 \% ; P=\text { not significant). }\end{array}$ & $3 / 4$ \\
\hline $\begin{array}{l}\text { Robles et al }{ }^{28} \text { (1998); } \\
\text { United States (Puerto } \\
\text { Rico); client-oriented } \\
\text { counseling }\end{array}$ & RCT; condom use (++) & $\begin{array}{l}\text { Out-of-treatment IDUs. I: 8-session } \\
\text { client-oriented approach targeting } \\
\text { perception of risk and motivation. } \\
\text { C: } 2 \text {-session standard care including } \\
\text { HIV test counseling. } \mathrm{N}=1016 \text { men } \\
\text { at baseline (numbers of men in each } \\
\text { group not reported). Follow-up: } 6 \text { mo. }\end{array}$ & $\begin{array}{l}\text { I group more likely to report condom use during } \\
\text { vaginal sex (OR, } 1.97 ; 95 \% \mathrm{CI}, 1.18 \text { to } 3.29) \text {. } \\
\text { Participants with a steady partner were less likely } \\
\text { to report condom use during vaginal sex at } \\
\text { follow-up (OR, } 0.50 ; 95 \% \mathrm{CI}, 0.28 \text { to } 0.90) \text {. } \\
\text { Participants reporting STI and those who were HIV } \\
\text { positive were more likely to use condoms during } \\
\text { vaginal sex (OR, } 26.89 ; 95 \% \mathrm{Cl}, 1.99 \text { to } 364.20 \\
\text { and OR, } 4.86 ; 95 \% \mathrm{CI}, 2.43 \text { to } 9.71 \text {, respectively). }\end{array}$ & $2 / 4$ \\
\hline \multicolumn{5}{|c|}{ Workers } \\
\hline $\begin{array}{l}\text { Jackson et al }{ }^{23}(1997) \text {; } \\
\text { Kenya; on-site clinic } \\
\text { offering range of } \\
\text { services }\end{array}$ & $\begin{array}{l}\text { Prospective, } 1 \text { group; } \\
\text { STI }(+), \text { condom use } \\
(-), \text { number of sex } \\
\text { partners }(+) \\
\text { (extramarital sex) }\end{array}$ & $\begin{array}{l}\text { Trucking workers in Mombasa. I: on-site } \\
\text { clinic providing counseling, HIV } \\
\text { testing, skills in condom negotiation, } \\
\text { demonstration and promotion, and } \\
\text { HIV/STI risk reduction. Supply of free } \\
\text { condoms. N = 856. Follow-up: every } \\
\text { 3-16 mo. }\end{array}$ & $\begin{array}{l}\text { The percentage reporting extramarital sex decreased } \\
\text { from } 49 \% \text { during the first quarter to } 36 \% \text { during } \\
\text { the last quarter of follow-up }(P<.001) \text {. No } \\
\text { differences in condom use. Decrease in incidence } \\
\text { of observed STI from } 34 \text { cases per } 100 \\
\text { patient-years during the first quarter to } 10 \text { cases } \\
\text { per } 100 \text { patient-years during the last quarter of } \\
\text { follow-up }(P=.001) \text {. }\end{array}$ & $3 / 5$ \\
\hline $\begin{array}{l}\text { Hearst et al }{ }^{19}(1999) ; \\
\text { Brazil; worksite-based } \\
\text { AIDS prevention } \\
\text { program }\end{array}$ & $\begin{array}{l}\text { Prospective, } 1 \text { group; } \\
\text { condom use }(+) \text {, } \\
\text { number of sex } \\
\text { partners }(+) \text {, } \\
\text { knowledge of AIDS } \\
\text { (-), attitudes toward } \\
\text { condoms }(-)\end{array}$ & $\begin{array}{l}\text { Male port workers. I: contact with peer } \\
\text { outreach workers, individually and } \\
\text { in groups, to discuss HIV/AIDS. } \\
\text { Free condoms. } \mathrm{N}=395 \text {. Follow-up: } \\
1 \text { and } 2 \mathrm{y} \text {. }\end{array}$ & $\begin{array}{l}\text { Significant drop in risk behavior after the I (at } 2 \mathrm{y} \text { ): } \\
\text { decrease in the number of nonprimary sex partners } \\
\text { (from a mean } 0.61 \text { to } 0.47 ; P=.02) \text { and increase in } \\
\text { consistent condom use }(21 \% \text { to } 12 \% ; P<.001) \text {. } \\
\text { Unprotected sex with casual partners decreased by } \\
64 \% \text { in } 2 \text { y }(P=.001) \text {. No effect of the I on } \\
\text { knowledge of AIDS and attitudes toward condoms. }\end{array}$ & $2 / 5$ \\
\hline $\begin{array}{l}\text { Leonard et al }{ }^{24}(2000) \text {; } \\
\text { Senegal; } \\
\text { Peer-education } \\
\text { worksite HIV/STI } \\
\text { prevention }\end{array}$ & $\begin{array}{l}\text { Prospective, } 1 \text { group; } \\
\text { number of sex } \\
\text { partners }(-) \text {, condom } \\
\text { use }(+), \text { HIV } \\
\text { knowledge }(+) \text {, } \\
\text { self-efficacy for using } \\
\text { condoms }(+)\end{array}$ & $\begin{array}{l}\text { Male trucking workers. I: contact with } \\
\text { peer workers to discuss HIV/STI. } \\
\text { Condom use skills, communication } \\
\text { skills taught. } \mathrm{N}=260 \text {. Follow up: } 2 \text { y. }\end{array}$ & $\begin{array}{l}\text { Significant increase in condom use at } 2 \text { y after I } \\
(88 \%) \text { from baseline }(52 \%)(P<.01) \text {. At follow-up, } \\
1.9 \% \text { reported having sex with a CSW in past } \\
\text { month compared with } 8.2 \% \text { at baseline }(P<.05) \text {, } \\
\text { but this was not substantiated by CSWs. } \\
\text { Knowledge of HIV reached } 100 \% \text { of men at } \\
\text { follow-up }(P<.01) \text {. Self-efficacy for condom use } \\
\text { increased for all items }(P<.01) \text {. }\end{array}$ & $2 / 5$ \\
\hline
\end{tabular}


Table 3. Characteristics, Results, and Assessed Quality of Included Sources* (cont)

\begin{tabular}{|c|c|c|c|c|}
\hline Source; Country; I & Design; Outcomes & Description & Results & $\begin{array}{c}\text { Assessed } \\
\text { Quality }^{16}\end{array}$ \\
\hline \multicolumn{5}{|c|}{ STI Clinics } \\
\hline $\begin{array}{l}\text { Boyer et } \text { al }^{31}(1997) \text {; } \\
\text { United States } \\
\text { (California); } \\
\text { cognitive-behavioral } \\
\text { skills-building I to } \\
\text { prevent STI in high-risk } \\
\text { heterosexual adults }\end{array}$ & $\begin{array}{l}\text { RCT; STI }(-) \text {, condom } \\
\text { use }(++), \text { number of } \\
\text { partners }(+)\end{array}$ & $\begin{array}{l}\text { People attending STI clinic. I: } 4 \text { individual } \\
\text { multiple-component sessions based } \\
\text { on decision-making and } \\
\text { communication skills to modify sexual } \\
\text { behavior: } n=69 \text { men (58\%). C: } 1 \\
\text { standardized risk-reduction counseling } \\
\text { session: } n=111 \text { men (75\%). } \\
\text { Follow-up: } 3 \text { and } 5 \text { mo, } 6 \text { mo (STI). }\end{array}$ & $\begin{array}{l}\text { No difference in new/probable STI at } 6 \text { mo ( } 13 \% \text { vs } \\
11 \% ; P>.20) \text {. At } 3 \text { mo, the I group showed } \\
\text { greater increase in percentage of time they used } \\
\text { condoms }(56.8 \% \text { vs } 42.3 \% ; P<.05) \text {. At } 5 \text { mo, } \\
\text { the mean number of sexual partners without use } \\
\text { of condoms was lower in the I group ( } 0.6 \text { vs } 0.9 \text {; } \\
P<.01 \text { ). }\end{array}$ & $4 / 4$ \\
\hline $\begin{array}{l}\text { Bentley et } \mathrm{al}^{21} \text { (1998); } \\
\text { India; HIV testing, } \\
\text { counseling, and } \\
\text { condom promotion }\end{array}$ & $\begin{array}{l}\text { Prospective, } 1 \text { group; } \\
\text { STI (+), condom use } \\
(+) \text {, number of sex } \\
\text { partners (-) } \\
\text { (continued visits to } \\
\text { CSWs), knowledge of } \\
\text { AIDS (+) }\end{array}$ & $\begin{array}{l}\text { Heterosexual men attending STI clinics. } \\
\text { I: individual counseling on } \\
\text { transmission, risk behaviors, } \\
\text { prevention, demonstration of condom } \\
\text { use. } \mathrm{N}=1628 \text {. Follow-up: every } 3 \text { mo } \\
\text { until } 24 \text { mo. }\end{array}$ & $\begin{array}{l}\text { Men who used condoms and who reported having } \\
\text { sex with fewer partners had the lowest prevalent } \\
\text { STI rate ( } 22 \% ; P=.001) \text {. After other factors } \\
\text { controlled for, the level of consistent condom use } \\
\text { with CSWs increased: men at } 24 \text { mo were } 4.7 \\
\text { times more likely to use condoms every time } \\
(P<.001) \text {. Among the } 56 \% \text { of men with a history } \\
\text { of STI, the likelihood of visiting CSWs had } \\
\text { increased at follow-up, with men at } 24 \text { mo } \\
3.2 \text { times more likely to visit CSWs ( } P<.001) \text {. } \\
\text { Knowledge of AIDS increased from } 45 \% \text { knowing } \\
\text { that condoms could prevent transmission of HIV } \\
\text { to } 89 \% \text { at } 6 \text { mo to } 96 \% \text { at } 24 \text { mo. }\end{array}$ & $3 / 5$ \\
\hline $\begin{array}{l}\text { Branson et al }{ }^{32} \text { (1998); } \\
\text { United States (Texas); } \\
\text { group counseling vs } \\
\text { standard } \\
\text { HIV-prevention } \\
\text { counseling }\end{array}$ & $\begin{array}{l}\text { RCT; new STI }(-) \text {, } \\
\text { condom use }(++) \text {, } \\
\text { number of sex } \\
\text { partners }(++)\end{array}$ & $\begin{array}{l}\text { STI clinic attendees. I: } 5 \text { sessions on } \\
\text { information, motivation, and } \\
\text { behavioral skills. C: } 2 \text { sessions of } \\
\text { standard HIV prevention counseling. } \\
\text { I: } n=289 ; C: \text { : } n=254 \text { (men). } \\
\text { Follow-up: } 2,6,9 \text {, and } 12 \text { mo. }\end{array}$ & $\begin{array}{l}\text { No group difference in new infections of gonorrhea, } \\
\text { chlamydia, or syphilis ( } 19 \% \text { vs } 19.3 \% ; P>.15) \text {. } \\
\text { Percentage using a condom from baseline to } \\
12 \text { mo: I, } 23 \% \text { vs } 48 \% ; C, 30 \% \text { vs } 49 \% ; P=.09 \text {. } \\
\text { No difference in percentage change reporting } \\
>1 \text { partner: I, } 42 \% \text { vs } 26 \% ; C, 42 \% \text { vs } 22 \% \text {; } \\
P=.21 \text {. }\end{array}$ & $3 / 4$ \\
\hline $\begin{array}{l}\text { Kalichman et al }{ }^{34} \text { (1999); } \\
\text { United States (Georgia); } \\
\text { (1) group motivational } \\
\text { sessions; (2) HIV } \\
\text { education sessions }\end{array}$ & $\begin{array}{l}\text { RCT; condom use }(++), \\
\text { talking to sex partner } \\
\text { about AIDS }(+) \text { and } \\
\text { about condoms }(+), \\
\text { planning to practice } \\
\text { safe sex (+), } \\
\text { knowledge (-), } \\
\text { attitudes (-), } \\
\text { intention to use } \\
\text { condoms }(-)\end{array}$ & $\begin{array}{l}\text { Inner-city black heterosexuals at STI } \\
\text { clinic. I: motivational sessions. C: } 2 \\
\text { HIV education sessions. I: } n=60 \text {; } \\
\text { C: } n=57 \text { (baseline). Follow-up: } \\
\text { post-I and } 3 \text { and } 6 \text { mo }\end{array}$ & $\begin{array}{l}\text { At } 3 \text { mo, more I group participants said that they } \\
\text { used condoms almost every time they had sex } \\
(69 \% \text { vs } 47 \% ; P=.02 \text { ) and that they had talked } \\
\text { with a sex partner about AIDS ( } 92 \% \text { vs } 70 \% \text {; } \\
P=.01 \text { ), and they reported lower rates of } \\
\text { unprotected vaginal sex (mean }=1.8 \text { vs } 7.4 \text {; } \\
P<.05 \text { ) and more use of condoms during } \\
\text { intercourse ( } 71 \% \text { vs } 54 \% ; P<.05) \text {. At } 6 \text { mo, I } \\
\text { group participants were more likely to discuss } \\
\text { condoms ( } 86 \% \text { vs } 68 \% ; P<.05 \text { ) and plan safer } \\
\text { sex ( } 93 \% \text { vs } 74 \% ; P=.01 \text { ). No differences in } \\
\text { knowledge, attitudes, or intentions. }\end{array}$ & $3 / 4$ \\
\hline $\begin{array}{l}\text { Cohen et al }{ }^{29}(1992) ; \\
\text { United States } \\
\text { (California); brief I } \\
\text { programs to promote } \\
\text { condom use among } \\
\text { patients in inner-city STI } \\
\text { clinics }\end{array}$ & RCT; new STI (+) & $\begin{array}{l}\text { Urban STI clinic attendees. I1: skills } \\
\text { approach. I2: social influence } \\
\text { approach: demonstrated condom use } \\
\text { and then focused on social/erotic } \\
\text { aspects of condom use. I3: condom } \\
\text { distribution through } 23 \text { local } \\
\text { businesses. C: no I. I1: } n=128, \text { I2: } \\
n=124, \text { I3: } n=137 \text { men. C: } 164 \text { men } \\
\text { (baseline). Follow-up: medical data at } \\
6 \text { to } 9 \text { mo. }\end{array}$ & $\begin{array}{l}\text { Fewer known reinfections in men exposed to the } \\
\text { condom skills }(\mathrm{I}=12.8 \% \text { vs } \mathrm{C}=87.2 \% \text { ) and } \\
\text { social influence ( } \mathrm{I}=12.5 \% \text { vs } \mathrm{C}=86.2 \% \text { ) than in } \\
\text { male } \mathrm{C} \text { participants (RR, } 0.43 ; 95 \% \mathrm{Cl}, 0.19 \text { to } \\
0.97 \text { and } \mathrm{RR}, 0.42 ; 95 \% \mathrm{CI}, 0.20 \text { to } 0.86 \text {, } \\
\text { respectively). }\end{array}$ & $2 / 4$ \\
\hline $\begin{array}{l}\text { National Institute of Mental } \\
\text { Health }{ }^{30} \text { (1998); United } \\
\text { States (7 sites in } \\
\text { California, Georgia, } \\
\text { Massachusetts, New } \\
\text { Jersey, New York, and } \\
\text { Wisconsin); behavioral I } \\
\text { to reduce HIV risk } \\
\text { behaviors }\end{array}$ & $\begin{array}{l}\text { RCT; new STI }(+) \text {, } \\
\text { unprotected sex }(+) \text {, } \\
\text { condom use }(+), \\
\text { STI symptoms }(+)\end{array}$ & $\begin{array}{l}\text { Low-income men (and women) at high } \\
\text { risk for HIV. I: seven 90- to 120-min } \\
\text { HIV risk-reduction group discussion } \\
\text { sessions, twice weekly. } \\
\text { C: } 1 \text {-h AIDS education session. } \\
\text { I: } n=783 \text { men; C: } n=781 \text { men. } \\
\text { Follow-up: } 3,6 \text {, and } 12 \text { mo. }\end{array}$ & $\begin{array}{l}\text { I group reported fewer unprotected sexual acts } \\
\text { (11.3 vs } 15.7) \text {, higher condom use }(0.64 \text { vs } \\
0.52) \text {, more consistent condom use }(45.1 \% \text { vs } \\
36.9 \%) \text { through } 12 \text { mo of follow-up (all } P<.01) \text {. } \\
\text { I group was less likely to report STI symptoms } \\
\text { (12.5 vs } 17.5 ; P<.05 \text { ) and had lower incidence of } \\
\text { gonorrhea based on chart review (3.6 vs } 6.4 \text {; } \\
P<.05 \text { ). }\end{array}$ & $2 / 4$ \\
\hline $\begin{array}{l}\text { O'Donnell et al }{ }^{36} \text { (1995); } \\
\text { United States (New } \\
\text { York); video-based } \\
\text { educational Is on } \\
\text { condom acquisition } \\
\text { among men (and } \\
\text { women) }\end{array}$ & $\begin{array}{l}\text { RCT; redeeming } \\
\text { coupons for free } \\
\text { condoms (+) } \\
\text { (intention to use } \\
\text { condoms) }\end{array}$ & $\begin{array}{l}\text { Hispanic and African American STI clinic } \\
\text { attendees. I1: video plus group skills- } \\
\text { building session. I2: video alone. } \\
\text { C: standard STI prevention } \\
\text { information. } \mathrm{N}=1033 \text { men (numbers } \\
\text { of men by group not reported). } \\
\text { Follow-up: post-I. }\end{array}$ & $\begin{array}{l}\text { Men exposed to both video and group session more } \\
\text { likely to redeem coupons (OR, } 1.28 ; 95 \% \mathrm{Cl} \text {, } \\
1.17 \text { to } 1.40) \text {. }\end{array}$ & $2 / 4$ \\
\hline
\end{tabular}


Table 3. Characteristics, Results, and Assessed Quality of Included Sources* (cont)

\begin{tabular}{|c|c|c|c|c|}
\hline Author (Year); Country; I & Design; Outcomes & Description & Results & $\begin{array}{c}\text { Assessed } \\
\text { Quality }^{16}\end{array}$ \\
\hline $\begin{array}{l}\text { Solomon and DeJong } \\
\text { (1989) (study 1); } \\
\text { United States } \\
\text { (Massachusetts); } \\
\text { Soap-opera style } \\
\text { videotape }\end{array}$ & $\begin{array}{l}\text { RCT; attitudes toward } \\
\text { condoms }(+) \text {, strategies } \\
\text { for persuading partners } \\
\text { to use condoms }(+)\end{array}$ & $\begin{array}{l}\text { Minority, at-risk STI clinic attendees. } \\
\text { Attitudes/strategies I: video portraying } \\
\text { acceptable, normative condom } \\
\text { bahavior. C: no I. I: } n=42 \text { men, } \\
\text { C: } n=42 \text { men (baseline). Follow-up: } \\
\text { post-I. }\end{array}$ & $\begin{array}{l}\text { I group scored higher on knowledge about need } \\
\text { and use of condoms (11.1 vs } 7.9 ; 95 \% \mathrm{Cl} \text { of } \\
\text { difference, } 2.7 \text { to } 3.7 \text { ). Mean attitude score } \\
\text { higher for the I group (difference in means, } 1.7 \text {; } \\
95 \% \mathrm{Cl} \text { of difference, } 1.0 \text { to } 2.4 \text { ). I group cited } \\
\text { more strategies for persuading partners to use } \\
\text { condoms (difference in means, } 0.5 ; 95 \% \mathrm{Cl} \text { of } \\
\text { difference, } 0.2 \text { to } 0.8 \text { ). }\end{array}$ & $2 / 4$ \\
\hline $\begin{array}{l}\text { Solomon and DeJong } \\
\text { (1989) (study 2); } \\
\text { United States } \\
\text { (Massachusetts); } \\
\text { soap-opera style } \\
\text { videotape }\end{array}$ & $\begin{array}{l}\text { RCT; redeeming coupons } \\
\text { for free condoms }(+) \\
\text { (intentions), knowledge } \\
\text { of condoms }(+)\end{array}$ & $\begin{array}{l}\text { Minority, at-risk STI clinic attendees. } \\
\text { I: video. C: no I. I: } n=71 \text { men; } \\
\text { C: } n=75 \text { men (baseline). Follow-up: } \\
\text { after I (not stated how many } \\
\text { days/weeks). }\end{array}$ & $\begin{array}{l}\text { Video group redeemed significantly more coupons } \\
\text { ( } 1.0 \text { vs } 0.8 \text {; difference in means, } 0.20 ; 95 \% \mathrm{Cl} \\
\text { of difference, } 0.02 \text { to } 0.38 \text { ). }\end{array}$ & $2 / 4$ \\
\hline $\begin{array}{l}\text { Wagstaff et al }{ }^{33}(1999) \\
\text { United States } \\
\text { (Pennsylvania); } \\
\text { educational sessions }\end{array}$ & $\begin{array}{l}\text { RCT; new STI (++), } \\
\text { condom use, risk } \\
\text { perception }\end{array}$ & $\begin{array}{l}\text { Black men. I1: individual education } \\
\text { session. I2: educational video. C: } \\
\text { standard care and education program. } \\
\mathrm{N}=562 \text {. (No experimental effects, so } \\
\text { results analyzed as a cohort, and } \\
\text { numbers per group not reported). } \\
\text { Follow-up: post-I and } 1 \text { and } 6 \text { mo. }\end{array}$ & $\begin{array}{l}\text { Rates of gonorrhea lower at } 6 \text {-mo follow-up than } \\
\text { at baseline ( } 34 \% \text { vs } 35 \%) \text {, chlamydia ( } 15 \% \text { vs } \\
23 \%) \text {, and herpes (3.2\% vs 3.7\%). P values not } \\
\text { reported. }\end{array}$ & $2 / 4$ \\
\hline \multicolumn{5}{|c|}{ Students } \\
\hline $\begin{array}{l}\text { Stanton et al }{ }^{39} \text { (1998); } \\
\text { Namibia; HIV } \\
\text { risk-reduction I among } \\
\text { Namibian adolescents }\end{array}$ & $\begin{array}{l}\text { RCT; condom use (+), } \\
\text { intentions to use } \\
\text { condoms (-), } \\
\text { communication skills } \\
\text { (+), self-efficacy for } \\
\text { condom use }(+)\end{array}$ & $\begin{array}{l}\text { Namibian students, aged } 15-18 \text { y. I: } 14 \\
\text { educational sessions in } \\
\text { communication and decision-making } \\
\text { skills. C: delayed controls. I: } n=262 \text {; } \\
\text { C: } n=253 \text {. Follow-up: } 2,6 \text {, and } \\
12 \text { mo. }\end{array}$ & $\begin{array}{l}\text { Among subjects who initiated sex during } \\
\text { follow-up, I group more likely to use condoms } \\
(18 \% \text { vs } 10 \% ; P<.05) \text {. No group difference in } \\
\text { condom intentions at } 12 \text { mo ( } 89 \% \text { vs } 88 \% \text {; } \\
P>.05) \text {. At } 6 \text { mo, I group more likely to } \\
\text { discuss sexual history and risk behavior with } \\
\text { partners ( } 70 \% \text { vs } 50 \% ; P<.05) \text {. I group had } \\
\text { higher self-efficacy of finding condoms when } \\
\text { needed ( } 94 \% \text { vs } 90 \% ; P<.05) \text {. }\end{array}$ & $4 / 4$ \\
\hline $\begin{array}{l}\text { Wight et al }{ }^{41} \text { (in press); } \\
\text { Scotland; theoretically } \\
\text { based teacher-delivered } \\
\text { sex education program }\end{array}$ & $\begin{array}{l}\text { RCT; condom use }(-) \text {, } \\
\text { quality of sexual } \\
\text { relationships }(+) \text {, sexual } \\
\text { health knowledge }(+)\end{array}$ & $\begin{array}{l}\text { School students. I: } 20 \text { sessions over } 2 \text { y } \\
\text { covering negotiation and condom use } \\
\text { skills. C: standard sex education. I: } \\
n=1299 ; \text { : } n=1437 \text { (male students } \\
\text { at } 6 \text { mo). Follow-up: } 6 \text { mo. }\end{array}$ & $\begin{array}{l}\text { No difference in condom use (mean } 3.80 \text { vs mean } \\
3.79 \text {; difference, } 0.01 ; 95 \% \mathrm{Cl} \text { for difference, } \\
-0.17 \text { to } 0.19 \text { ) or in unprotected sex at } \\
\text { follow-up ( } 13.98 \% \text { vs } 13.88 \% \text {; difference, } 0.09 \text {; } \\
95 \% \mathrm{Cl} \text { for difference, }-2.10 \text { to } 2.29 \text { ). I male } \\
\text { students less likely to report regret of first } \\
\text { sexual intercourse ( } 9.55 \% \text { vs } 19.41 \% ; \\
\text { difference, }-9.86 ; 95 \% \mathrm{Cl} \text { for difference, }-18.70 \\
\text { to }-1.04) \text {, and greater knowledge of sexual } \\
\text { health at baseline ( } 4.35 \% \text { vs } 3.66 \% \text {; difference, } \\
0.69 \% ; 95 \% \mathrm{Cl} \text { for difference, } 0.20 \% \text { to } \\
1.19 \%) \text {. }\end{array}$ & $3 / 4$ \\
\hline $\begin{array}{l}\text { Antunes et al }{ }^{40}(1997) \\
\text { Brazil; HIV/AIDS } \\
\text { prevention workshop }\end{array}$ & $\begin{array}{l}\text { RCT; condom use (-), } \\
\text { partner communication } \\
\text { about sex and AIDS (-) }\end{array}$ & $\begin{array}{l}\text { Heterosexual adult students. } \\
\text { I: } 4 \text { sessions of group discussions } \\
\text { on sexual norms, social roles, AIDS } \\
\text { risk, HIV transmission; condom use } \\
\text { practiced, role-playing. C: wait list. } \\
\text { l: } n=69 ; \text { C: } n=72 \text {. Follow-up: } 6 \text { mo. }\end{array}$ & $\begin{array}{l}\text { At } 6 \text { mo, no group differences in sexual behavior } \\
\text { (no means reported; } P>.05 \text { ) or partner } \\
\text { communication (mean }=17.29 \text { vs } 18.55 \\
P>.05 \text { ) }\end{array}$ & $2 / 4$ \\
\hline $\begin{array}{l}\text { Jemmott et } \text { al }^{38}(1992) \text {; } \\
\text { United States } \\
\text { (Pennsylvania); } \\
\text { informational and } \\
\text { skills-building session }\end{array}$ & $\begin{array}{l}\text { RCT; condom use }(+) \text {, } \\
\text { number of sexual } \\
\text { partners }(+), \text { knowledge } \\
\text { of AIDS }(+) \text {, attitudes } \\
(+), \text { intentions regarding } \\
\text { risky sexual behavior }(+)\end{array}$ & $\begin{array}{l}\text { Black male youths. I: } 5 \text {-h informational } \\
\text { and skills-building session. C: } 5 \text {-h } \\
\text { matched careers session. I: } n=85 ; C \text { : } \\
n=72 \text { Follow-up: post-I and } 3 \text { mo. }\end{array}$ & $\begin{array}{l}\text { I immediately improved AIDS knowledge ( } 46.23 \\
\text { vs } 40.37 ; P<.001) \text {, attitudes ( } 3.02 \text { vs } 3.48 ; \\
P<.004) \text {, and intentions ( } 2.83 \text { vs } 3.40 ; \\
P<.001) \text {. Also improved at } 3 \text { mo: knowledge } \\
\text { (47.20 vs } 44.40 ; P<.003) \text {, intentions ( } 2.87 \text { vs } \\
3.30 ; P<.007) \text {, and behavior ( }-0.12 \text { vs } 0.24 ; \\
P<.01) \text {. I reduced sexual intercourse frequency } \\
(2.15 \text { d vs } 5.48 \mathrm{~d} ; P<.05) \text {, number of partners } \\
\text { (0.85 vs } 1.79 ; P<.05) \text {, and frequency without a } \\
\text { condom }(0.64 \text { vs } 2.38 ; P<.05) \text {. }\end{array}$ & $2 / 4$ \\
\hline $\begin{array}{l}\text { St Lawrence et al }{ }^{37} \\
\text { (1995); United States } \\
\text { (Mississippi); } \\
\text { behavioral skills } \\
\text { training I for black } \\
\text { adolescents }\end{array}$ & $\begin{array}{l}\text { RCT; unprotected vaginal } \\
\text { sex (++), condom use } \\
(-) \text {, AIDS knowledge }(-) \text {, } \\
\text { attitudes to condoms } \\
(-) \text {, self-efficacy }(-), \\
\text { response efficacy }(-), \\
\text { personalization of risk } \\
\text { (-) }\end{array}$ & $\begin{array}{l}\text { Low-income black adolescents. } \\
\text { I: } 8 \text {-session skills training. C: } 12 \text {-h } \\
\text { educational session. } \mathrm{N}=69 \text { male } \\
\text { adolescents ( } 28 \% \text { of sample). } \\
\text { Numbers of male participants in each } \\
\text { group not reported. Follow-up: } 12 \text { mo. }\end{array}$ & $\begin{array}{l}\text { I group lowered frequency of unprotected vaginal } \\
\text { intercourse compared with } C(P<.05) \text {. No } \\
\text { group differences in condom-protected vaginal } \\
\text { sex, AIDS knowledge, condom attitudes, } \\
\text { self-efficacy, response efficacy, or risk } \\
\text { personalization. }\end{array}$ & $2 / 4$ \\
\hline
\end{tabular}


Table 3. Characteristics, Results, and Assessed Quality of Included Sources* (cont)

\begin{tabular}{|c|c|c|c|c|}
\hline Author (Year); Country; I & Design;Outcomes & Description & Results & $\begin{array}{l}\text { Assessed } \\
\text { Quality }{ }^{16}\end{array}$ \\
\hline $\begin{array}{l}\text { Sanderson }{ }^{42} \text { (1999); } \\
\text { United States (New } \\
\text { Jersey); HIV prevention } \\
\text { videos }\end{array}$ & $\begin{array}{l}\text { RCT; self-efficacy for eroticizing } \\
\text { condom use }(-) \text {, self-efficacy } \\
\text { for condom use in difficult } \\
\text { situations }(+)\end{array}$ & $\begin{array}{l}\text { University students (male and female). } \\
\text { I1: communication skills video. I2: } \\
\text { technical skills video. I3: combined } \\
\text { skills video. C: wait list. } \mathrm{N}=102 \\
\text { men at baseline (numbers of men } \\
\text { by group not reported). Follow-up: } \\
\text { post-I and } 3 \text { mo. }\end{array}$ & $\begin{array}{l}\text { Post-I, combined skills video group had } \\
\text { lower self-efficacy condom use in } \\
\text { difficult situations (mean }=3.99 \\
\text { compared with technical, mean }=4.24 \text {, } \\
\text { and communication, mean }=4.20 ; \\
P<.04 \text { ). No group differences on } \\
\text { self-efficacy for eroticizing condom use } \\
\text { (technical, communication, combined, } \\
\text { and C groups, mean }=3.74,3.76,3.71 \text {, } \\
\text { and } 3.89 \text {, respectively; } P>.05 \text { ). }\end{array}$ & $2 / 4$ \\
\hline \multicolumn{5}{|c|}{ Other Studies } \\
\hline $\begin{array}{l}\text { Vaz et al }{ }^{20} \text { (1996); } \\
\text { Mozambique; AIDS } \\
\text { education sessions }\end{array}$ & $\begin{array}{l}\text { Prospective, } 1 \text { group; AIDS } \\
\text { knowledge }(+)\end{array}$ & $\begin{array}{l}\text { Prisoners. I: } 3 \text { educational sessions led } \\
\text { by prisoners focusing on (1) the } \\
\text { impact of STI/AIDS in prisons; } \\
\text { (2) transmission; and (3) AIDS } \\
\text { prevention. Theater group presented } \\
\text { monthly shows. N = 300. } \\
\text { Follow-up: } 6 \text { mo. }\end{array}$ & $\begin{array}{l}\text { Increase in the proportion of correct } \\
\text { answers for AIDS knowledge questions } \\
(P<.01) \text {. }\end{array}$ & $4 / 5$ \\
\hline $\begin{array}{l}\text { Susser et al } 44 \text { (1998); } \\
\text { United States (New } \\
\text { York); I to reduce } \\
\text { sexual risk behaviors } \\
\text { among homeless } \\
\text { mentally ill men }\end{array}$ & $\begin{array}{l}\text { RCT; sexual risk index (+ at } 6 \\
\text { mo), VEE points for } \\
\text { unprotected sex, condom } \\
\text { use (+), number of sex } \\
\text { partners (+) }\end{array}$ & $\begin{array}{l}\text { Homeless men in a psychiatric } \\
\text { outreach program; l: } 15 \text { sessions } \\
\text { with storytelling, games, } \\
\text { role-playing, condom skills training. } \\
\text { C: } 2 \text { educational sessions and } \\
\text { condom use instructions. } \\
\text { l: } n=52 \text { ( } 23 \text { sexually active); } \\
\text { C: } n=45 \text { ( } 26 \text { sexually active). } \\
\text { Follow-up: } 6,12 \text {, and } 18 \text { mo. }\end{array}$ & $\begin{array}{l}\text { At } 6 \text { mo, VEE scores were higher in the } C \\
\text { group (3.1) than in the I group (1.0) } \\
(P<.01) \text {. Rate of protected sexual } \\
\text { episodes higher in I group ( } 0.73 \text { vs } \\
0.52 ; P=0.04) \text {. Percentage of high-risk } \\
\text { behavior lower in I group ( } 24 \% \text { vs } \\
58 \% ; P=.01) \text {. No significant group } \\
\text { differences at } 12 \text { or } 18 \text { mo. }\end{array}$ & $3 / 4$ \\
\hline $\begin{array}{l}\text { Celentano et } \mathrm{al}^{43}(1998) \text {; } \\
\text { Thailand; Mass } \\
\text { communication } \\
\text { campaign }\end{array}$ & $\begin{array}{l}\text { Prospective, } 2 \text { groups (cohorts) } \\
\text { at } 2 \text { different times; new STI } \\
(+)\end{array}$ & $\begin{array}{l}\text { Thai military men. I: mass } \\
\text { communication campaign to } \\
\text { promote awareness of avoiding } \\
\text { brothels, provision of condoms to } \\
\text { CSWs, condom use promotion. } \\
\text { I: } n=2147 \text { (1991 cohort); } \\
n=1669 \text { (1993 cohort). Follow-up: } \\
\text { up to } 24 \text { mo. }\end{array}$ & $\begin{array}{l}\text { Significant decline in STI incidence within } \\
1991 \text { cohort (18.6 per } 100 \\
\text { patient-years; } P<.0001) \text {; borderline } \\
\text { trend was observed within the } 1993 \\
\text { cohort (1.7 per } 100 \text { patient-years; } \\
P=.06 \text { ). Inconsistent condom use } \\
\text { significantly less common in } 1993 \text { than } \\
\text { in } 1991 \text { (1991, } 16 \% ; 1993,3 \% \text {; } \\
P<.001) \text {. }\end{array}$ & $3 / 5$ \\
\hline $\begin{array}{l}\text { Kalichman et al } 45 \text { (1997); } \\
\text { United States } \\
\text { (Wisconsin); } \\
\text { cognitive-behavioral } \\
\text { skills training in HIV } \\
\text { prevention paradigm }\end{array}$ & $\begin{array}{l}\text { RCT; condom use (-), number } \\
\text { of vaginal sex episodes (++), } \\
\text { strategies to reduce HIV } \\
\text { risk (++), intentions to } \\
\text { change risk behaviors }(++) \text {, } \\
\text { AIDS-related knowledge (++) }\end{array}$ & $\begin{array}{l}\text { Black men. I: cognitive-behavioral } \\
\text { skills training and condom use } \\
\text { practice. C: HIV risk education and } \\
\text { sensitization. I: } n=44 ; \text { C: } n=37 \text {. } \\
\text { Follow-up: } 2 \text { weeks post-I and } 3 \text { mo. }\end{array}$ & $\begin{array}{l}\text { No differences between groups were } \\
\text { reported on any variable. }\end{array}$ & $2 / 4$ \\
\hline
\end{tabular}

*I indicates intervention(s); C, control activity(ies); HIV, human immunodeficiency virus; plus sign in parentheses, significant I effect; double plus sign in parentheses, significant changes reported in I and C groups; minus sign in parentheses, no significant I effect; IDU, injecting drug user; RCT, randomized controlled trial; AIDS, acquired immunodeficiency syndrome; NS, not significant; OR, odds ratio; Cl, confidence interval; STI, sexually transmitted infection; CSW, commercial sex worker; RR, risk ratio; and VEE, vaginal episode equivalent.

characterized by their use of an RCT study design and their focus on US men. Only 1 intervention used a prospective uncontrolled design with a long follow-up period, and this was conducted in a non-US population. ${ }^{21}$ Most interventions with this group of men sought to reduce morbidity: 5 studies in this group aimed to decrease the incidence of STI. ${ }^{29-33}$ Two studies reported a significant decrease in STI incidence as a result of the intervention; these interventions involved a multiple-component motivation and skills approach to reducing the risk of
HIV. ${ }^{29,30}$ Two studies reported no decrease in STI incidence; these interventions also consisted of a multiplecomponent motivation and skills approach to reducing the risk of HIV. ${ }^{31,32}$ One study reported a decrease in STI in the intervention and control groups. ${ }^{33}$ This intervention involved an individually based AIDS education session, while the comparison and control groups consisted of either a video-based education session or the clinic's standard care and education.

Five interventions in STI clinics aimed to change men's sexual be- havior. ${ }^{21,30-32,34}$ One study reported significant intervention effects in the experimental group on unprotected sexual acts, condom use, and consistent condom use. ${ }^{30}$ In a study involving an individual counseling session providing education and condom use skills, condom use increased in this group of men. ${ }^{21}$ However, the number of sex partners did not decrease over the 2-year follow-up. Three studies reported an increase in condom use in men in the experimental and control groups. ${ }^{31,32,34}$ These interventions all consisted of motivational and skills-building components. In one 
study, men and women were divided into single-sex intervention groups $^{31}$; in another, men and women participated in mixed-sex sessions ${ }^{32}$; and in another study (exclusively with men), participants were placed into groups. $^{34}$

Four studies focusing on men in STI clinics sought to change social psychological outcomes. ${ }^{21,34-36}$ Intentions to use condoms increased in the 2 studies of video-based education interventions that aimed to change this behavior. ${ }^{35,36}$ In 1 study of a motivational and skills-based intervention, intentions to use condoms did not increase in the experimental group. ${ }^{34}$ Mixed results were found in the 2 studies addressing attitudes toward condoms, ${ }^{34,35}$ with one reporting a positive change in attitudes ${ }^{35}$ and the other reporting no change. ${ }^{34}$ Mixed results were also reported for knowledge of AIDS. One study of a counseling and skills-based intervention showed a positive intervention effect on knowledge, ${ }^{21}$ while another of a motivational and skills-based intervention reported no effect. ${ }^{34}$ Communication skills with sex partners about the risk of AIDS $^{34}$ and condom use $e^{34,35}$ showed a significant intervention effect in the 2 studies addressing these outcomes.

\section{Heterosexual Male Students}

Six moderate- to high-quality RCTs targeted heterosexual male students. $^{37-42}$ These studies aimed to change behavioral and social psychological outcomes; morbidity was not addressed. Four studies focused on younger school-aged men, ${ }^{37-39,41}$ one on university men, ${ }^{41}$ and 1 study targeted men attending night school. ${ }^{40}$ Two studies were conducted with non-US populations. ${ }^{39,40}$

The behavioral outcomes addressed were unprotected sex, ${ }^{37}$ condom use, ${ }^{37-41}$ and number of sex partners. ${ }^{38}$ In a behavioral skillstraining intervention, St Lawrence et $\mathrm{al}^{37}$ reported a decrease in the frequency of unprotected sex among men in the intervention and control groups. ${ }^{37}$ Significant intervention effects on condom use were reported in 2 studies, ${ }^{38,39}$ while 3 studies reported no effect. ${ }^{37,40,41}$ All 5 interventions aimed at increasing condom use included cognitive- behavioral and skills training components.

All 6 studies aimed to change social psychological outcomes. In the study to address intentions to use condoms, these intentions did not change, ${ }^{39}$ while in the study that sought to change intentions to engage in risky behavior, significant intervention effects were reported. ${ }^{38}$ Attitudes toward condoms did not change in one study, ${ }^{37}$ but attitudes toward risky behavior did in another. ${ }^{38}$ Increases in knowledge about sexual health ${ }^{41}$ and AIDS ${ }^{38}$ were successfully brought about by interventions that addressed these outcomes. However, knowledge of AIDS did not change in another intervention. ${ }^{37}$ Mixed results were also reported for changes in communicating about sex and AIDS with partners: intervention effect was reported in one study, ${ }^{39}$ but no change was reported in another. ${ }^{40}$ Selfefficacy for using condoms was found to increase in one study ${ }^{42}$ and not another. ${ }^{37}$ Each of these interventions was similar in that each involved behavioral skills training in condom use, decision making, and communication skills. Active learning, role-playing, and practicing of skills were all part of the interventions.

\section{Other Men}

Four studies involved different male populations..$^{20,43-45} \mathrm{~A}$ mass national communication campaign was conducted in Thailand, and its effectiveness was assessed through a prospective cohort study with men entering the Thai military over 2 periods. ${ }^{43}$ The authors reported a significant reduction in the incidence of new STIs over the course of the study. Two moderate-quality RCTs addressed behavioral outcomes. ${ }^{44,45}$ One RCT with homeless men with psychiatric diagnoses reported a positive intervention effect on the frequency of unprotected sex, condom use, and the number of sex partners of these men. ${ }^{44}$ Another RCT with African American men contacted through an outreach program reported no intervention effect on condom use. ${ }^{45}$ Both interventions consisted of multiple skillstraining sessions focusing on deci- sion making, condom use, and behavioral self-management.

Two studies aimed to change social psychological outcomes. In a cognitive-behavioral RCT, changes were reported in intentions to engage in risky behavior and AIDSrelated knowledge in the intervention and control groups. ${ }^{45}$ A significant increase in knowledge of AIDS was reported in a prospective uncontrolled study with male prisoners in Mozambique consisting of education sessions led by prisoner activists. $^{20}$

COMMENT

This is the first systematic review to assess the effectiveness of interventions to prevent STIs and HIV in heterosexual men-a heterogeneous population currently the focus of a concerted global effort to reduce the risk of STI for themselves and their partners, including the risk of HIV. ${ }^{1}$ We found 27 studies that met our inclusion criteria for a systematic review. We believe that this reflects the relatively new focus of interest in heterosexual men's sexual health. Until recently, the main focus on heterosexual men's reproductive and sexual health has been on attitudes toward contraception and family planning and men's roles in increasing the risk and vulnerability of their female sex partners, with relatively little effort concentrated on men's own sexual health concerns. ${ }^{11}$ Given that in many settings it is the behavior of the male partner that places women at increased risk of STI, including HIV, it is imperative to identify strategies and interventions that may work to decrease the burden of risk and disease among heterosexual men and their female partners.

Most of the studies identified through the present review were designed to evaluate behavioral and/or social psychological, rather than morbidity, outcomes. Most were undertaken in the United States, and thus do not reflect the global epidemiology of STIs and HIV in terms of incident and prevalent infections. Nonetheless, we believe that the review has highlighted the following points that will influence the design of future interventions and further research. 


\section{WHICH INTERVENTIONS WERE EFFECTIVE IN REDUCING THE BURDEN OF NEW DISEASE?}

Only a minority of studies were designed to evaluate the effectiveness of reducing the incidence of new STIs or HIV infection. Most interventions aimed to change social psychological or behavioral outcomes. Of the 8 interventions designed to reduce the incidence of new STIs (including HIV), 5 were deemed to be successful, 2 were not successful, and 1 gave equivocal results (reduction in incidence in the intervention and control groups). The 5 successful (but not necessarily high methodological quality) interventions were carried out among men in the workplace ( 1 study), men in the military ( 1 study), and men in STI clinics (3 studies). A variety of methods were used in these interventions, including on-site counseling and HIV testing at a trucking company with individual sessions for participating men; a mass communications (and multiple-sector) approach to risk reduction in Thailand; and multiple-component motivation and skills approaches in STI clinics. In the latter case, 2 further studies using similar intervention methods reported no decrease in STI incidence.

What do these results tell us about the effectiveness of interventions? First, it is important to examine the need for studies that use laboratory-diagnosed STI as the outcome of interest. If reduced incidence of HIV or other STI is to become the gold standard goal of intervention research, ${ }^{46}$ studies of social psychological programs will have to demonstrate empirically the relationship between cognitions and infection. Many psychological theories of behavior emphasize the role of attitudes, intentions, and selfefficacy in determining behavior. ${ }^{47-49}$ However, systematic reviews and meta-analyses of these theories have shown that the evidence supporting the role of social psychological variables in predicting behavior is often conflicting. ${ }^{50,51}$ It is possible that attitudes and intentions predict behavior, and that these may be important variables to include in interventions to prevent the spread of STIs and HIV.
In this review, only 1 study among heterosexual men was identified that made the connection between change in social psychological measures, behavior, and morbidity, but this study did not use an RCT design to assess efficacy. ${ }^{21}$

Second, there is no single intervention that can be identified as being more effective than others in reducing the incidence of STI and HIV in heterosexual men. This finding is presumably a reflection of the heterogeneity of the groups of men under study and the wide variety of different contexts in which interventions were being evaluated. Successful interventions ranged from localized to national responses, but all were resource intensive either in their execution or their measurement (eg, the 2 successful interventions in STI clinics were both RCTs). This carries important implications for those charged with program and intervention design-a single "cookie-cutter" approach is unlikely to be successful in any given setting. Moreover, achieving and recording the success of an intervention is a resource-intensive undertaking, especially if gold standard laboratory outcomes are to be measured.

\section{WHICH INTERVENTIONS WERE EFFECTIVE IN CHANGING MEN'S ATTITUDES AND BEHAVIORS?}

Most studies were designed to measure the effect of interventions to change social psychological and behavioral outcomes. As with the studies that aimed to decrease incident STI, there was no single method that could be identified as being effective in all situations aiming to change behaviors, increase knowledge, or measure an intention to change.

All 3 studies targeting heterosexual drug users in treatment reported success in increasing condom use, decreasing the number of reported sexual partners, and improvement in social psychological outcomes. These results are especially notable in a population often described as "difficult." In addition, 2 studies were carried out among drug users out of treatment programs, and one showed an in- crease in condom use (but in both the intervention and control groups). Again, no single intervention strategy could be identified as being consistently successful, partly because a variety of methods were used in all the interventions reviewed.

Among men in other clinicbased settings (STI clinics), a small number of interventions were found to improve behavioral outcomes. A variety of methods were used, but positive effects were noted as frequently in the control groups as in the intervention groups in most studies. Two studies using video-based education programs showed an increase in intention to use condoms, and a counseling and skills-based intervention showed a significant improvement in communication skills with partners. However, the results for all other social psychological outcomes were difficult to interpret, with studies showing no improvement in other indicators of attitudinal change.

Studies of interventions with men in the workplace all reported significant intervention effects on the men's sexual behavior and knowledge of HIV and STI. These interventions show that other study designs apart from RCTs can demonstrate changes in sexual behavior of high-risk men (eg, truckers who have frequent contact with commercial sex workers). The use of peer educators in interventions of longer duration seem to have been effective. Accuracy of self-reports can also be validated by secondary sources, as in the study by Leonard and colleagues. ${ }^{24}$

Similar results were found in studies among men in other settings-students, homeless men, and prisoners. Even when similar intervention methods were used, results were not consistent between one group of men and another. Interventions that showed a positive outcome in one setting produced equivocal results elsewhere. A wide variety of approaches were used throughout these interventions, including individual counseling, group counseling, mixed-sex counseling, single-sex counseling, and repeat and one-off sessions, with no evidence of effect size being related to the form that interventions took. 


\section{POTENTIAL BIASES OF THE REVIEW PROCESS}

Although we attempt herein to answer the question, "Which interventions are successful in reducing the transmission of STIs in heterosexual men?" most of the studies we evaluate do not include only heterosexual men. Interventions that included women along with heterosexual men were included if data on men were presented and analyzed separately from those on women or if men constituted $80 \%$ or more of the study population. While it is possible that the presence of women in mixed-sex intervention sessions may have influenced men's participation in these sessions,$^{52}$ without further empirical investigation it is difficult to determine this influence. We decided to include these studies to increase the number for review when it became apparent that few interventions exclusively addressed heterosexual men.

Many of the studies were small and reported statistically significant results, which suggests that there is a risk of publication bias (ie, that small studies with negative findings are under-represented in the present review). This may be because such studies have been rejected for publication or because researchers have not submitted them for publication. A formal test for publication bias has not been carried out given the heterogeneity of the study designs and the ongoing debate about the applicability of current statistical approaches to observational studies. ${ }^{53}$ However, the number of small studies with positive results is suggestive of publication bias, and the possibility remains that this review may, if anything, overestimate the effectiveness of interventions in this area.

\section{POTENTIAL BIASES OF THE STUDIES THEMSELVES}

Most of the studies were conducted in the United States, with just under half of these US-based studies conducted on racial and ethnic minority populations. While recognizing the importance of these results for STI and HIV control in the United States (and espe- cially given racial disparities in STI risk in the country), the findings cannot easily be transferred to the rest of the world, and especially to those countries currently experiencing the burden of incident and prevalent STIs and HIV infection. Several areas of the world are notable by their complete absence from the evidence base that we have reviewed: Eastern Europe and Central Asia (currently experiencing epidemics of HIV and other STIs) and Eastern Asia (home to a significant proportion of the world's population and under increasing threat of an HIV epidemic). In addition, other regions with a high burden of STIs and HIV disease or a potential burden of disease are represented by only 1 or 2 study results (eg, Africa and South Asia).

Most of the studies were undertaken among groups of men who were easily identifiable as being at risk of STIs and HIV (men attending STI clinics or injecting drug users). Other studies targeted very specific groups of men in the population: homeless men, students, and prisoners.

\section{CONCLUSIONS}

These potential biases suggest that caution should be used when interpreting the results. On the basis of the present review, it is not possible to prescribe the optimum approach to reduce the risk of transmission of STIs, including HIV, in heterosexual men. We identified relatively few methodologically rigorous studies (and even fewer with a gold standard biological indicator of behavior as the outcome) and no single consistently effective approach to reduce incident infections, change behavior, or change social psychological outcomes.

On a more optimistic note, however, it is worth remembering that although there were only 4 interventions that addressed most men in the population (who are not in clinic populations, not in education, or do not have an identifiable risk behavior), they all showed a positive behavioral intervention effect. These were interventions among men in the workplace or men joining the military after a nationwide mass media and structural intervention to reduce HIV risk in Thailand. This suggests that it is possible to reduce the burden of sexual risk and consequent ill health for men in the general population-the section of men likely to have the largest population-attributable risk for the burden of STI and HIV in men and women in many settings.

There is clearly a pressing need to identify successful programs to reduce HIV and STI transmission and morbidity among heterosexual men. However, it will only be possible to measure reduced incidence of HIV in heterosexual men in circumstances of high population prevalence of the infection. In other situations, including those where incident STIs are high, it may be necessary to use reductions in non-HIV morbidity as a proxy marker of program efficacy. There are problems in using bacterial infections such as Chlamydia trachomatis and Neisseria gonorrhoeae as indicators of risk for viral infections such as HIV because of biological differences in transmission dynamics, but they remain good markers of exposure to sexually transmitted pathogens. Also, transmission of these bacterial infections is prevented in the same way as HIV infections are prevented, notably through protected sex or sexual intercourse between uninfected partners.

The results of the present systematic review suggest that the following factors are important for future research agendas to prevent the spread of STIs and HIV in heterosexual men:

- Research needs to focus on morbidity outcomes (eg, incident infection) rather than only behavioral or social psychological outcomes.

- Interventions need to target heterosexual men or at least ensure that heterosexual men participate in single-sex intervention groups, and then evaluations can identify the approaches that are best suited to this population.

- More research needs to be carried out in regions of the world 
where rates of STI and HIV are high among heterosexual men (such as sub-Saharan Africa) and where they are increasing (such as Asia, Eastern Europe, and Central Asia).

- Studies other than RCTs can identify promising interventions; the effectiveness of these interventions requires further rigorous evaluation before widespread implementation.

- Studies should have the statistical power to demonstrate effectiveness, and this should be calculated prior to research being funded and implemented.

Accepted for publication April 24, 2002.

Corresponding author and reprints: Graham J. Hart, PhD, Medical Research Council Social and Public Health Sciences Unit, University of Glasgow, 4Lilybank Gardens, Glasgow G12 8RZ, Scotland (e-mail: graham@ mrc.msoc.gla.ac.uk).

\section{REFERENCES}

1. Joint United Nations Program on HIV/AIDS. Report on the Global HIV/AIDS Epidemic. Geneva, Switzerland: UNAIDS; 2000.

2. Mayaud P, Mosha F, Todd J, et al. Improved treatment services significantly reduce the prevalence of sexually transmitted diseases in rural Tanzania: results of a randomized controlled trial. AIDS. 1997;11:1873-1880.

3. Grosskurth H, Mosha F, Todd J, et al. A community trial of the impact of improved sexually transmitted disease treatment on the HIV epidemic in rural Tanzania, II: baseline survey results. AIDS. 1995;9:927-934.

4. Jones RB, Wasserheit JN. Introduction to the biology and natural history of sexually transmitted diseases. In: Wasserheit JN, Aral SO, Holmes KK, eds. Research Issues in Human Behavior and Sexually Transmitted Diseases in the AIDS Era. Washington, DC: American Society for Microbiology; 1991:11-37.

5. Harlap S, Kost K, Forrest J. Preventing Pregnancy, Protecting Health: A New Look at Birth Control Choices in the United States. New York, NY: Alan Guttmacher Institute; 1991.

6. Aral S. Heterosexual transmission of HIV: the role of other sexually transmitted infections and behavior and its epidemiology, prevention and control. Annu Rev Public Health. 1993;14:451467.

7. Greene ME, Biddlecom AE. Absent and Problematic Men: Demographic Accounts of Male Reproductive Roles. New York, NY: Population Council; 1997.

8. Moses S, Muia E, Bradley JE, et al. Sexual behavior in Kenya: implications for sexually transmitted disease transmission and control. Soc Sci Med. 1994;39:1649-1656.

9. Oakley A, Fullerton D, Holland J. Behavioural in- terventions for HIV/AIDs prevention. AIDS. 1995; 9:479-486.

10. Weinhardt L, Carey M, Johnson B, Bickham N. Effects of HIV counseling and testing on sexual risk behavior: a meta-analysis review of published research, 1985-1997. Am J Public Health. 1999;89: 1397-1405.

11. Hawkes S, Hart GJ. Reproductive health: men's roles and men's rights. In Jejeebhoy S, Keonig M, Shah I, eds. Undertaking Community Surveys of Gynaecological Morbidity. Cambridge, England: Cambridge University Press. In press.

12. Forste R, Morgan J. How relationships of US men affect contraceptive use and efforts to prevent sexually transmitted diseases. Fam Plann Perspect. 1998;30:56-62.

13. Wasserheit JN. Epidemiological synergy: interrelationships between human immunodeficiency virus infection and other sexually transmitted diseases. Sex Transm Dis. 1992;19:61-77.

14. Grosskurth H, Mosha F, Todd J, et al. Impact of improved treatment of sexually transmitted diseases on HIV infection in rural Tanzania: randomised controlled trial. Lancet. 1995;346: 530-536.

15. Landis JR, Koch GG. The measurement of observer agreement for categorical data. Biometrics. 1977;33:159-174.

16. Jadad AR, Moore A, Carroll D, et al. Assessing the quality of reports of randomized clinical trials: is blinding necessary? Control Clin Trials. 1996;17: 1-12.

17. Deeks J, Glanville J, Sheldon T. Undertaking Systematic Reviews of Effectiveness: CRD Guidelines for Those Carrying Out or Commissioning Reviews. York, England: NHS Centre for Reviews and Dissemination, University of York; 1996. CRD Report 4.

18. Stroup DF, Berlin JA, Morton SC, et al, for the Meta-analysis of Observational Studies in Epidemiology (MOOSE) Group. Meta-analysis of Observational Studies in Epidemiology: a proposal for reporting. JAMA. 2000;283:20082012.

19. Hearst N, Lacerda R, Gravato N, Sid Hudes E, Stall R. Reducing AIDS risk among port workers in Santos, Brazil. Am J Public Health. 1999;89: 76-78.

20. Vaz RG, Gloyd S, Trindade R. The effects of peer education on STI and AIDS knowledge among prisoners in Mozambique. Int J STD AIDS. 1996;7: 51-54.

21. Bentley ME, Spratt K, Shepherd ME, et al. HIV testing and counseling among men attending sexually transmitted disease clinics in Pune, India: changes in condom use and sexual behavior over time. AIDS. 1998;12:1869-1877.

22. Calsyn DA, Meinecke C, Saxon A, Stanton V. Risk reduction in sexual behavior: a condom giveaway program in a drug abuse treatment clinic. Am J Public Health. 1992;82:1536-1538.

23. Jackson DJ, Rakwar JP, Richardson BA, et al. Decreased incidence of sexually transmitted diseases among trucking company workers in Kenya: results of a behavioural risk-reduction programme. AIDS. 1997;11:903-909.

24. Leonard L, Ndiaye I, Kapadia A, et al. HIV prevention among male clients of female sex workers in Kaolack, Senegal: results of a peer education program. AIDS Educ Prev. 2000;12:21-37.

25. Baker A, Kochan N, Dixon J, Heather N, Wodak A. Controlled evaluation of a brief intervention for HIV prevention among injecting drug users not in treatment. AIDS Care. 1994;6:559-570.

26. Malow R, West J, Corrigan S, Pena J, Cunning- ham S. Outcome of psychoeducation for HIV risk reduction. AIDS Educ Prev. 1994:6:113-125.

27. Cottler LB, Compton WM, Ben Abdallah A, et al. Peer-delivered interventions reduce HIV risk behaviors among out-of-treatment drug abusers. Public Health Rep. 1998;113(suppl):31-41.

28. Robles RR, Marrero CA, Matos TD, et al. Factors associated with changes in sex behavior among drug users in Puerto Rico. AIDS Care. 1998;10: 329-338.

29. Cohen DA, Dent C, MacKinnon D, Hahn G. Condoms for men, not women: results of brief promotion programs. Sex Transm Dis. 1992;19: 245-250.

30. National Institute of Mental Health (NIMH). The NIMH multisite HIV prevention trial: reducing HIV sexual risk behavior. Science. 1998;280:18891894.

31. Boyer CB, Barrett DC, Peterman TA, Bolan G. Sexually transmitted disease (STD) and HIV risk in heterosexual adults attending a public STD clinic: evaluation of a randomized controlled behavioral risk-reduction intervention. AIDS. 1997;11: 359-367.

32. Branson BM, Peterman TA, Cannon R0, Ransom R, Zaidi AA. Group counseling to prevent sexually transmitted disease and HIV: a randomized controlled trial. Sex Transm Dis. 1998;25:553560

33. Wagstaff DA, Delamater JD, Havens KK. Subsequent infection among adolescent AfricanAmerican males attending a sexually transmitted disease clinic. J Adolesc Health. 1999;25:217226.

34. Kalichman SC, Cherry C, Browne-Spearling F. Effectiveness of a video-based motivational skills-building HIV risk-reduction intervention for inner-city African American men. J Consult Clin Psychol. 1999;67:959-966.

35. Solomon MZ, DeJong W. Preventing AIDS and other STDs through condom promotion: a patient education intervention. Am J Public Health. 1989;79:453-458

36. O'Donnell LN, San Doval A, Duran R, O'Donnell C. Video-based sexually transmitted disease patient education: its impact on condom acquisition. Am J Public Health. 1995;85:817-822.

37. St Lawrence JS, Brasfield TL, Jefferson KW, Alleyne E, O'Brannon RE III, Shirley A. Cognitivebehavioral intervention to reduce African American adolescents' risk for HIV infection. J Consult Clin Psychol. 1995;63:221-237.

38. Jemmott JB III, Jemmott LS, Fong GT. Reductions in HIV risk-associated sexual behaviors among black male adolescents: effects of an AIDS prevention intervention. Am J Public Health. 1992; 82:372-377.

39. Stanton BF, LiXi, Kahihuata J, et al. Increased protected sex and abstinence among Namibian youth following a HIV risk-reduction intervention: a randomized, longitudinal study. AIDS. 1998;12:24732480.

40. Antunes MC, Stall RD, Paiva V, et al. Evaluating an AIDS sexual risk reduction program for young adults in public night schools in Sao Paulo, Brazil. AIDS. 1997;11:S121-S127.

41. Wight D, Raab GM, Henderson M, et al. The limits of teacher-delivered sex education: interim behavioral outcomes from a randomized trial. BMJ. In press.

42. Sanderson CA. Role of relationship context in influencing college students' responsiveness to HIV prevention videos. Health Psychol. 1999;18: 295-300.

43. Celentano DD, Nelson KE, Lyles CM, et al. De-

(REPRINTED) $\overline{\text { ARCH INTERN MED/VOL 162, SEP 9, } 2002 \quad \text { WWW.ARCHINTERNMED.COM }}$ 
creasing evidence of HIV and sexually transmitted diseases in young Thai men: evidence for success of the HIV/AIDS control and prevention program. AIDS. 1998;12:F29-F36.

44. Susser E, Valencia E, Berkman A, et al. Human immunodeficiency virus sexual risk reduction in homeless men with mental illness. Arch Gen Psychiatry. 1998;55:266-272.

45. Kalichman SC, Rompa D, Coley B. Lack of positive outcomes from a cognitive-behavioral HIV and AIDS prevention intervention for inner-city men: Iessons from a controlled pilot study. AIDS Educ Prev. 1997;9:299-313.
46. Aral SO, Peterman TA. Do we know the effectiveness of behavioural interventions? Lancet. 1998; 351(suppl 3):33-36.

47. Fishbein M, Ajzen I. Belief, Attitude, Intention and Behavior: An Introduction to Theory and Research. Reading, Mass: Addison-Wesley; 1975.

48. Ajzen I. Attitudes, Personality and Behavior. Milton Keynes, England: Open University Press; 1988.

49. Bandura A. Self-efficacy: toward a unifying theory of behavioral change. Psychol Rev. 1977;84:191215.

50. Hausenblas HA, Carron AV, Mack DE. Application of the theories of reasoned action and planned behavior to exercise behavior: a meta-analysis. Int J Sport Psychol. 1997;19:36-51.

51. Ouellette J, Wood W. Habit and intention in everyday life: the multiple process by which past behavior predicts future behavior. Psychol Bull. 1998;124:54-74.

52. Hodgins DC, el-Guebaly N, Addington J. Treatment of substance abusers: single or mixed gender programs? Addiction. 1997;92:805-812.

53. Sterne JAC, Egger M, Davey Smith G. Systematic reviews in health care: investigating and dealing with publication and other biases in meta-analysis. BMJ. 2001;323:101-105.

\title{
CME Announcement
}

\section{CME Hiatus: July Through December 2002}

CME from JAMA/Archives will be suspended between July and December 2002. Beginning in early 2003, we will offer a new online CME program that will provide many enhancements:

- Article-specific questions

- Hypertext links from questions to the relevant content

- Online CME questionnaire

- Printable CME certificates and ability to access total CME credits

We apologize for the interruption in CME and hope that you will enjoy the improved online features that will be available in early 2003.

\author{
(REPRINTED) $\overline{\text { ARCH INTERN MED/VOL 162, SEP 9, } 2002 \quad \text { WWW.ARCHINTERNMED.COM }}$
}

1830

ㄷ2002 American Medical Association. All rights reserved. 